\title{
High harmonic generation in crystalline silicon irradiated by an intense ultrashort laser pulse
}

\author{
Tzveta Apostolova ${ }^{1,2, a}$ and Boyan Obreshkov ${ }^{1}$ \\ 1 Institute for Nuclear Research and Nuclear Energy, Bulgarian Academy of Sciences, Tsarigradsko Chausse 72,1784 Sofia, \\ Bulgaria \\ 2 Institute for Advanced Physical Studies, New Bulgarian University, 1618 Sofia, Bulgaria
}

Received 2 July 2021 / Accepted 6 September 2021 / Published online 6 October 2021

(C) The Author(s) 2021

\begin{abstract}
We investigate the high harmonic generation in bulk silicon irradiated by intense near-infrared laser pulses with pulse duration $\leq 100 \mathrm{fs}$. For peak field strength of the applied laser is below $1 \mathrm{~V} / \AA$, the spectral intensity of the emitted harmonics follows the prediction of perturbative nonlinear optics-the frequency comb consists of a series of discrete peaks at odd harmonic orders. For a pulse duration longer than 30 fs and peak laser field strength exceeding $1 \mathrm{~V} / \AA$, non-perturbative effects and generation of even order harmonics occur. The appearance of even harmonics is due to optical rectification of the transmitted pulse, which includes weak quasi-DC component with electric field as low as $3 \mathrm{~V} / \mu \mathrm{m}$. In the strong coupling regime, when the peak field strength inside vacuum exceeds $1.5 \mathrm{~V} / \AA$, the laser creates dense breakdown plasma of electron-hole pairs, which in turn results in severe spectral broadening of the transmitted pulse. The harmonic spectrum superimposes onto a continuous background, the spectral width of individual harmonics is substantially broadened, and their central wavelength undergoes a blue shift that covers the spacing between adjacent harmonic orders.
\end{abstract}

\section{Introduction}

High harmonic generation (HHG) in solids has been demonstrated experimentally [1-3] and is associated with sub-cycle dynamics of electron-hole pairs $[1,2,4-$ 7]. Experiments were conducted in the recent years that show the possibility of using solid-state HHG for alloptical reconstruction of band structure [8] and carrier dynamics [9,10], for light-driven electronics [7,11-14], for generation of terahertz radiation sources [4], and for ultrafast dielectric optical switching [15].

In inversion symmetric materials such as silicon, even order harmonic generation does not occur in long wavelength approximation. However, inversion symmetric materials can generate even harmonics from higherorder nonlinear responses [16]. Harmonic spectrum extending from the visible to deep ultraviolet spectral range generated in silicon crystal irradiated by a 100 fs laser pulse with mid-infrared wavelength of $3.5 \mu \mathrm{m}$ was observed recently [17]. The HHG spectra were consistently interpreted within the semi-classical threestep re-collision model [18], including nonlinear photoionization, the kinetic energy gain of the freed electron through their acceleration in the laser electric field, followed by the radiative re-combination of the electron with its parent ion near zero-crossings of the laser electric field, and finally resulting in emission of ionizing

\footnotetext{
a e-mail:

tzveta.apostolova@nbu.bg author)

(corresponding
}

XUV irradiation. It was demonstrated that HHG in silicon is affected by weak DC electric fields as low as $30 \mathrm{~V} / \mu \mathrm{m}$, which allows generation of even order harmonics. An array of monopole nano-antennas fabricated on a 500-nm-thick single-crystal Si film was used in Ref. [19] to produce plasmon-assisted HHG directly from the supporting crystalline silicon substrate. The mechanism of even order harmonic generation was associated with field enhancement near the edge of the nano-antenna, causing electrons propagating towards it to experience a stronger field compared to the one experienced by electrons traveling away from it. This is also reflected in a different phase of the rapidly oscillating dipole associated with HHG during each two successive half-cycles of the driving field. Thus, the occurrence of even harmonics was attributed to asymmetry of the laser field. The mechanism of even-order harmonic generation in ionic crystals was also discussed in Ref. [20,21] and shown to be a result of the phase of the complex-valued transition dipole moment connecting valence to conduction bands.

In this work, we present numerical results of HHG in bulk silicon irradiated by linearly polarized intense laser pulses of $800 \mathrm{~nm}$ wavelength. By varying the pulse duration in the range 10-100 fs and the peak laser intensity from $10^{11}$ to $10^{14} \mathrm{~W} / \mathrm{cm}^{2}$, we have calculated the ultrafast photo-induced electric currents in bulk silicon associated to HHG. In Sect. 2, we present basic theory and discuss numerical results related to the time evolution of the electric field of the transmitted pulse, the 
HHG spectra and harmonic efficiency from bulk silicon. Section 3 includes our main conclusions, unless otherwise stated we use atomic units $\left(e=\hbar=m_{e}=1\right)$.

\section{Numerical results and discussion}

\subsection{Basic theory}

Detailed description of the static band structure of silicon, the theoretical approach and methodology can be found in Refs. [22-25]. In this approach, valence electrons in Si were assumed to respond as non-interacting particles to the total electric field, which is a superposition of the time-dependent electric field of the transmitted pulse and the static screened electric field of the substrate ionic cores. The externally applied laser electric field is parameterized by a Gaussian function $\mathbf{E}_{\text {ext }}(t)=\mathbf{e} \exp \left[-\ln (4)\left(t-t_{0}\right)^{2} / \tau^{2}\right] F \cos \omega_{\mathrm{L}} t$, where $\mathbf{e}$ is the laser polarization vector, $\omega_{\mathrm{L}}$ is the laser oscillation frequency, $\tau$ is the pulse length, $t_{0}$ designates the position of the pulse peak, and $F$ is the electric field strength related to the peak laser intensity by $I=F^{2}$. In response to the applied field, the movement of electrons results in transiently polarized solid with an induced electric field $\mathbf{E}_{\text {ind }}=-4 \pi \mathbf{P}$ that superimposes onto the applied one to determine the electric field of the transmitted pulse $\mathbf{E}=\mathbf{E}_{\text {ext }}+\mathbf{E}_{\text {ind }}$ inside the bulk. The macroscopic polarization is related to the transient photo-induced electric current by $\mathbf{J}(t)=\mathrm{d} \mathbf{P} / \mathrm{d} t$ which can be written as:

$$
\mathbf{J}(t)=\sum_{v=1}^{4} \int_{B Z} \frac{\mathrm{d}^{3} \mathbf{k}}{4 \pi^{3}}\langle v \mathbf{k}(t)|\mathbf{p}+\mathbf{k}+\mathbf{A}(t)| v \mathbf{k}(t)\rangle,
$$

where $\mathbf{p}=-i \nabla_{\mathbf{r}}$ is the momentum operator, $\mathbf{A}(t)$ is the macroscopic vector potential related to the pulsed electric field by $\mathbf{E}(t)=-\partial_{t} \mathbf{A}, v$ labels the four initially occupied valence electron orbitals of silicon and $\mathbf{k}$ is the crystal momentum, which extends over the first Brillouin zone. The HHG spectra and the harmonic yield are obtained from the Fourier transform of the transient current projected onto the laser polarization direction

$$
Y(\omega)=\left|\int \mathrm{d} t e^{i \omega t} \mathbf{J}(t) \cdot \mathbf{e}\right|
$$

The time-dependent lattice-periodic Bloch states $|v \mathbf{k}(t)\rangle$ in Eq. 1 are obtained by numerically solving the Schrödinger equation $i \partial_{t}|v \mathbf{k}(t)\rangle=H(\mathbf{k}, t)|v \mathbf{k}(t)\rangle$ with the Hamiltonian of a Bloch electron

$$
H(\mathbf{k}, t)=\frac{1}{2}[\mathbf{p}+\mathbf{k}+\mathbf{A}(t)]^{2}+V_{\mathrm{e}-\text { ion }}(\mathbf{r})
$$

where $V_{\text {e-ion }}(\mathbf{r})$ is the static screened electron-ion pseudo-potential for valence electrons [26], which exhibits the periodicity of the diamond lattice. Because the diamond lattice has an inversion symmetry, the pseudo- potential $V_{\text {e-ion }}(\mathbf{r})=V_{\text {e-ion }}(-\mathbf{r})$ does not change under spatial inversion, thus according to the perturbative nonlinear optics, the generation of even harmonics is forbidden, e.g. the second-order nonlinear susceptibility vanishes identically for $\mathrm{Si}$, such that optical rectification and second-harmonic generation are forbidden processes. Therefore, generation of even-order harmonics from bulk Si subjected to strong laser fields is also indicative of a breakdown of perturbation theory approach.

\subsection{Dependence of HHG on the laser intensity and pulse duration}

\subsubsection{Results for 30 fs pulse}

Figure 1a, c and e shows the time evolution of the electric field transmitted in bulk $\mathrm{Si}$ irradiated by $30 \mathrm{fs}$ pulsed laser with near-infrared wavelength $800 \mathrm{~nm}$ (photon energy $\hbar \omega_{L}=1.55 \mathrm{eV}$ ). In the low intensity regime with $I=3 \times 10^{13} \mathrm{~W} / \mathrm{cm}^{2}$, shown in Fig. 1a, dielectric response of $\mathrm{Si}$ is exhibited-the electric field follows the oscillations of the applied laser with $\mathbf{E}=$ $\mathbf{E}_{\text {ext }} / \epsilon_{0}$, where $\epsilon_{0}=12$ is the static dielectric constant of Si. When the laser intensity is increased to $7 \times 10^{13} \mathrm{~W} / \mathrm{cm}^{2}$, Fig. 1c, the field strength inside the bulk reaches $0.2 \mathrm{~V} / \AA$ at the pulse peak, the delayed response of the polarization manifests in time delay of the peak of the transmitted pulse relative to the applied laser. For high intensity with $9 \times 10^{13} \mathrm{~W} / \mathrm{cm}^{2}$ shown in Fig. 1e, the laser has ionized large fraction of valence electrons and created free electrons in the conduction band. The response of the free charge carriers to the applied laser causes rapid decrease in the refractive index of $\mathrm{Si}$, such that the transmitted pulse becomes subject to self-phase modulation in the breakdown plasma. The temporal profile of the pulse is strongly distorted: the field strength exceeds $0.2 \mathrm{~V} / \AA$ already on the rising edge of the pulse and grows rapidly in time until it reaches its peak value of $0.4 \mathrm{~V} / \AA$ in the trailing edge of the applied laser. Next, a steep edge is formed in the back of the pulse due to screening of the laser field by conduction electrons, the laser frequency becomes unstable and up-shifts, i.e. a positive frequency chirp is exhibited. This frequency up-chirp causes severe spectral broadening of the transmitted pulse and affects the mechanism for HHG in the strong-coupling regime.

The associated HHG spectra are shown in Fig. 1b, d and f. For the lowest intensity shown in Fig. 1b, the spectrum consists of isolated peaks at odd harmonics extending up to the 15th order. Similar trend is observed in the intermediate intensity regime, shown in Fig. 1d, the frequency comb now extends to higher orders, and cut-off is exhibited at the 21st order. For the highest peak intensity shown in Fig. 1e, harmonic peaks are broadened and superimpose onto a continuous background, the harmonic peaks undergo an uniform blue shift $\Delta \omega \approx 0.1 \omega_{L}$, which makes evident that harmonics are mainly emitted at the back of the pulse when the driving frequency is up-chirped. 

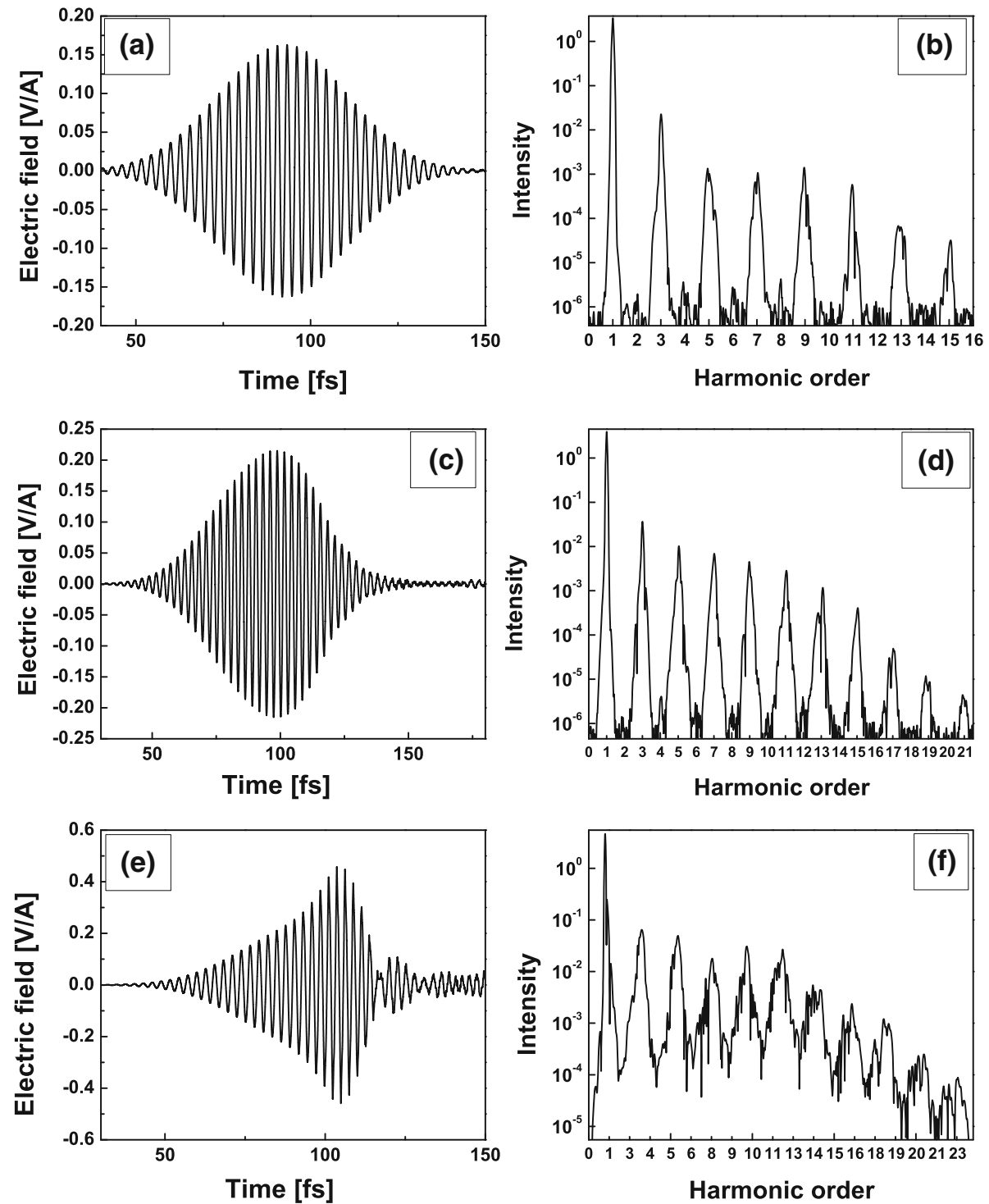

Fig. 1 Time evolution of the pulsed total electric field (in $\mathrm{V} / \AA$ ) in bulk silicon for $\tau=30$ fs. The laser intensity at the pulse peak is $3 \times 10^{13} \mathrm{~W} / \mathrm{cm}^{2}$ in $(\mathbf{a}), 7 \times 10^{13} \mathrm{~W} / \mathrm{cm}^{2}$ in $(\mathbf{c})$ and is $9 \times 10^{13} \mathrm{~W} / \mathrm{cm}^{2}$ in (e). The laser is linearly polarized along the [001] direction, and the laser wavelength is $800 \mathrm{~nm}$. The corresponding harmonic spectra are shown in (b), (d), (f)

\subsubsection{Results for 60 and 90 fs pulses}

The time evolution of the electric field of the transmitted pulse, when the duration of the applied laser pulse is increased to $60 \mathrm{fs}$, is shown in Fig. 2a, c and e. The dielectric response of $\mathrm{Si}$ is exhibited in the relatively low laser intensity regime with $I=3 \times 10^{13} \mathrm{~W} / \mathrm{cm}^{2}$ when the temporal profile of the transmitted pulse is weakly distorted by the laser-matter interaction and exhibits nearly Gaussian profile, cf. Fig. 2a. For the increased peak intensity $5 \times 10^{13} \mathrm{~W} / \mathrm{cm}^{2}$, shown in Fig. 2c, the build-up of plasma of photo-excited carriers results in time delay of the pulse peak relative to the peak of the applied laser, similar to the result for the $30 \mathrm{fs}$ pulse (cf. also Fig. 1d). The temporal profile of the transmitted pulse strongly deteriorates in the strong- coupling regime corresponding to peak laser intensity $7 \times 10^{13} \mathrm{~W} / \mathrm{cm}^{2}$, cf. Fig. 2e.

The corresponding harmonic spectra are shown in Fig. 2b, d and f. For the lowest intensity shown in Fig. 2b, the frequency comb now consists of both odd and even order harmonics, the high-energy cut-off is positioned at the 15th harmonic order. The spectral intensity of even order harmonics is lower than the intensity of odd order ones. A plateau region of the harmonic spectrum is exhibited in the intermediate intensity regime in Fig. 2d, and the cut-off energy for HHG increases to 21st order. The spectral intensity of even order harmonics now becomes comparable to the intensity of odd harmonics. The emergence of even order harmonics is a result of optical rectification of the transmitted pulse and generation of low-frequency $(\mathrm{THz}) \mathrm{com}-$ 

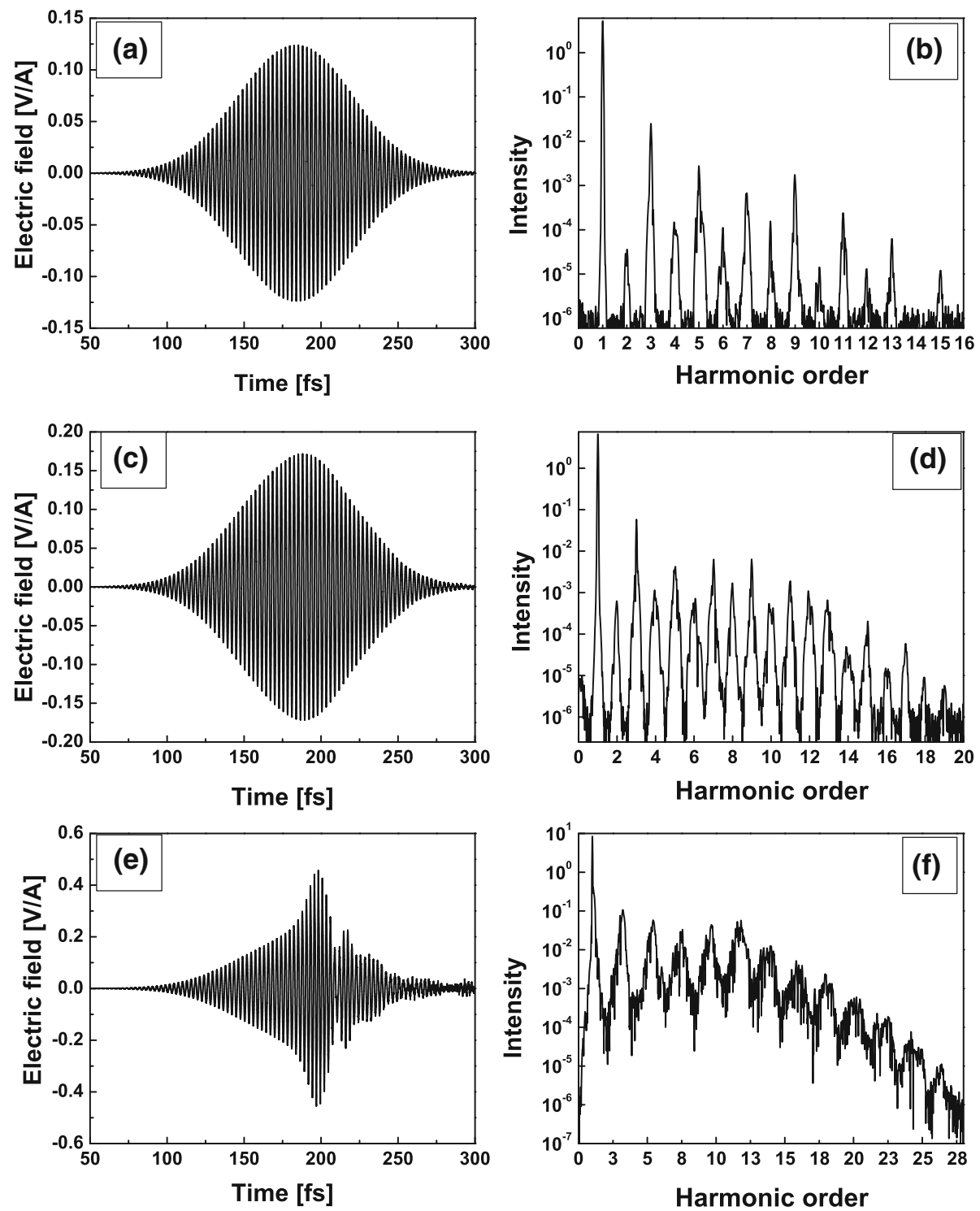

Fig. 2 Time evolution of the pulsed total electric field (in $\mathrm{V} / \AA$ ) in bulk silicon for $\tau=60$ fs. The laser intensity at the pulse peak is $3 \times 10^{13} \mathrm{~W} / \mathrm{cm}^{2}$ in (a), $5 \times 10^{13} \mathrm{~W} / \mathrm{cm}^{2}$ in $(\mathbf{c})$ and is $7 \times 10^{13} \mathrm{~W} / \mathrm{cm}^{2}$ in (e). The laser is linearly polarized along the [001] crystal direction, and the laser wavelength is $800 \mathrm{~nm}$. The corresponding harmonic spectra are shown in (b), (d), (f)

ponent of the pulsed electric field, shown in Fig. 3. A DC-like electric field as low as $2 \mathrm{~V} / \mu \mathrm{m}$ is generated after the pulse peak, when the oscillating laser electric field is still strong. When a weak quasi-DC component is present, the phase of the rapidly oscillating dipole associated with HHG is different during the first and during the second half-cycle of oscillation of the driving laser, which enables the emission of even harmonics in bulk silicon. The emission of even order harmonics is weakly dependent on the specific band structure of silicon and is a primary consequence of the symmetry of the dynamical system under space- and time-reversal. To display this effect schematically, we apply the semiclassical re-collision model $[20,21]$. The Fourier compo- nents of the HHG current can be written as:

$$
\begin{array}{r}
J(\omega)=\int_{\mathrm{BZ}} \frac{\mathrm{d}^{3} \mathbf{k}}{4 \pi^{3}} \int \mathrm{d} t e^{-i \omega t} \mathbf{e} \cdot \mathbf{p}_{v c}(\mathbf{k}(t)) \\
\int^{t} \mathrm{~d} t^{\prime} \mathbf{E}\left(t^{\prime}\right) \cdot \mathbf{d}_{c v}\left(\mathbf{k}\left(t^{\prime}\right)\right) e^{-i S\left(\mathbf{k}, t, t^{\prime}\right)}
\end{array}
$$

where $\mathbf{k}(t)=\mathbf{k}+\mathbf{A}(t)$ is the shifted crystal momentum, the matrix element of the momentum in a static Bloch state basis is $\mathbf{p}_{c v}=\langle c \mathbf{k}|\mathbf{p}| v \mathbf{k}\rangle$, the transition dipole moment is $\mathbf{d}_{c v}=\mathbf{p}_{c v} / i \Delta$, here $\Delta$ is the band gap energy $(\Delta(\mathbf{k})=\Delta(-\mathbf{k})$ as a consequence of time reversal symmetry) and $S\left(\mathbf{k}, t, t^{\prime}\right)=\int_{t^{\prime}}^{t} \mathrm{~d} \tau \Delta(\mathbf{k}(\tau))$ is 


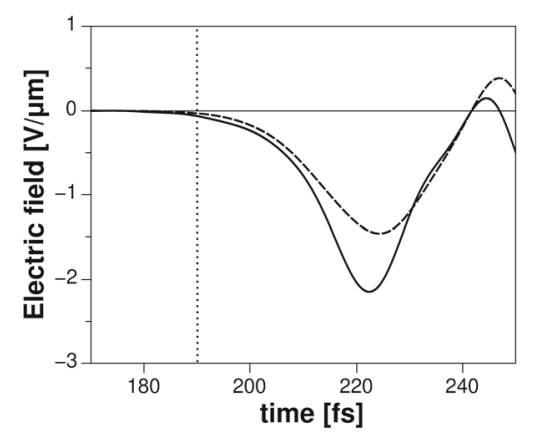

Fig. 3 Time evolution of the quasi-DC field (in $\mathrm{V} / \mu \mathrm{m}$ ) generated in bulk $\mathrm{Si}$, after irradiation with $60 \mathrm{fs}$ laser pulse. The peak laser intensity of the driving pulse is $4 \times 10^{13} \mathrm{~W} / \mathrm{cm}^{2}$ (dashed line) and $5 \times 10^{13} \mathrm{~W} / \mathrm{cm}^{2}$ (solid line), the vertical dotted line indicates the position of the pulse peak. The applied laser is linearly polarized along the [001] crystal direction, and the laser wavelength is $800 \mathrm{~nm}$

the quasiclassical action for an electron-hole pair propagating in the laser field. For simplicity, we choose the crystal momentum $\mathbf{k}=k \mathbf{e}$ to point along the laser polarization direction, introduce the scalar function $d_{c v}(k)=\mathbf{e} \cdot \mathbf{d}_{c v}(k \mathbf{e})$ and denote the saddle points of the action by $\left(k_{s}, t_{r}, t_{i}\right)$, where $t_{i}$ and $t_{r}$ can be interpreted as the (complex) times of ionization and re-combination of an electron-hole pair, respectively. During the time interval between ionization and photo-recombination, electron-hole pairs gain kinetic energy in the laser field, which is converted into harmonic photon energy at the time of recombination. The contribution of an isolated saddle point to the current is proportional to

$$
p_{v c}\left(k_{r}\right) d_{c v}\left(k_{i}\right) E\left(t_{i}\right) e^{-i \omega t_{r}} e^{-i S\left(k_{s}, t_{r}, t_{i}\right)} .
$$

where $k_{i}=k_{s}+A\left(t_{i}\right)$ and $k_{r}=k_{s}+A\left(t_{r}\right)$ designate the shifted crystal momenta at the time of ionization and recombination, respectively. During the next halfcycle of the oscillating electric field, when $E\left(t+\pi / \omega_{L}\right) \approx$ $-E(t)$, the saddle point with $\left(-k_{s}, t_{r}+\pi / \omega_{L}, t_{i}+\pi / \omega_{L}\right)$ gives an equal in magnitude contribution to the current, and the coherent sum of these two amplitudes is

$$
\begin{aligned}
& p_{v c}\left(k_{r}\right) d_{c v}\left(k_{i}\right) E\left(t_{i}\right)\left[e^{-i S\left(k_{s}, t_{r}, t_{i}\right)}\right. \\
& \left.\quad-e^{-i S\left(-k_{s}, t_{r}+\pi / \omega_{L}, t_{i}+\pi / \omega_{L}\right)} e^{-i \pi \omega / \omega_{L}}\right]
\end{aligned}
$$

where we have used that for the inversion-symmetric diamond lattice $d_{c v}(k)= \pm d_{c v}(-k)$. In the presence of weak DC field of strength $E_{0}$, the action can be written as:

$$
\begin{aligned}
S\left(k, t, t^{\prime}\right)= & \int \mathrm{d} \tau \Delta\left(k(\tau)+E_{0} \tau\right) \approx S_{0}\left(k, t, t^{\prime}\right) \\
& +E_{0} \int_{t^{\prime}}^{t} \mathrm{~d} \tau \tau v(k(\tau))
\end{aligned}
$$

here $v(k)=\partial \Delta / \partial k$ is the group velocity of the wavepacket. Because $S_{0}\left(k_{s}, t_{r}, t_{i}\right)=S_{0}\left(-k_{s}, t_{r}+\pi / \omega_{L}, t_{i}+\right.$ $\left.\pi / \omega_{L}\right)$, and since $v(k)=-v(-k)$, the contribution of these two saddle points to the $n$-th harmonic (after setting $\left.\omega / \omega_{L}=n\right)$ becomes

$$
p_{v c}\left(k_{r}\right) d_{c v}\left(k_{i}\right) F\left(t_{i}\right) e^{-i S_{0}\left(k_{s}, t_{i}, t_{r}\right)}\left[e^{-i \Delta S}-e^{i \Delta S} e^{-i n \pi}\right]
$$

where $\Delta S\left(k, t, t^{\prime}\right)=E_{0} \int_{t^{\prime}}^{t} \mathrm{~d} \tau \tau v(k(\tau))$ is the phase shift induced by the DC field. The HHG yield is thus proportional to the factor $|\sin (n \pi / 2+\Delta S)|$. If there is no DC field, only odd-order harmonics are emitted, $\sin (n \pi)=0$; otherwise, generation of even-order harmonics is enabled because of the phase shift $\Delta S$ accumulated during propagation in the quasi-DC field.

The HHG spectrum in the breakdown regime is also shown in Fig. 2d for the 60 fs pulse. The emergence of broad quasi-continuous spectrum is qualitatively similar to the HHG spectrum for the $30 \mathrm{fs}$ pulse. The results for $90 \mathrm{fs}$ pulse duration are summarized in Fig. $4 \mathrm{a}-\mathrm{f}$. Similar to the result for 60 fs laser pulse, Fig. $4 \mathrm{~b}$ and $\mathrm{d}$ displays the yield of both even and odd harmonics for peak laser intensities $3 \times 10^{13} \mathrm{~W} / \mathrm{cm}^{2}$ and $4.5 \times 10^{13}$ $\mathrm{W} / \mathrm{cm}^{2}$.

\subsubsection{Conversion efficiency}

Figures 5, 6 and 7 summarize the dependence of the harmonic yield $Y_{n}$ on the peak field strength $F$ for 30, 60 and 90 fs laser pulses. The harmonic yield is related to the spectral intensity of harmonics by $I_{n}=Y_{n}^{2}$, and the conversion efficiency is $I_{n} / I_{1}$, where $I_{1}$ is the intensity of the fundamental harmonic. The dependence on the laser polarization vector direction is also shown: in Figs. 5a, 6a and 7a the laser is linearly polarized along the [001] crystal direction, and the laser polarization vector points in the [111] direction in Figs. 5b, 6b and $7 \mathrm{~b}$.

As seen from the figures, the conversion efficiency for HHG from silicon is low and does not exceed $10^{-4}$. The following regimes of HHG could be distinguished: I. Perturbative regime with $F \leq 1 \mathrm{~V} / \AA$, the scaling law $Y_{n} \sim F^{n}$ is exhibited, where $n$ labels the harmonic order. Only odd harmonics are emitted in bulk silicon. For the [001] polarization, the intensities of the 7th and the 9th harmonic cross, subsequently an inversion in their efficiencies occurs, while for the [111] polarization direction crossing of the 9 th with 11 th harmonic is observed. It may be worth noting that inversion of the generation efficiencies of 2 nd and 3rd harmonics from gold surfaces irradiated by near-infrared femtosecond pulse was experimentally observed in Ref. [27]. However, the mechanisms for HHG in bulk solids and metal surfaces are different.

II. Non-perturbative regime (with $F>1 \mathrm{~V} / \AA$ ): The HHG spectrum extends to higher orders as the field strength increases. The intensities of above band gap harmonics with $n \geq 5$ are of comparable spectral strength and run parallel to each other. In this regime, even-order harmonics are generated for 60 and 90 fs laser pulses. Initially, the yield of even harmonics is 

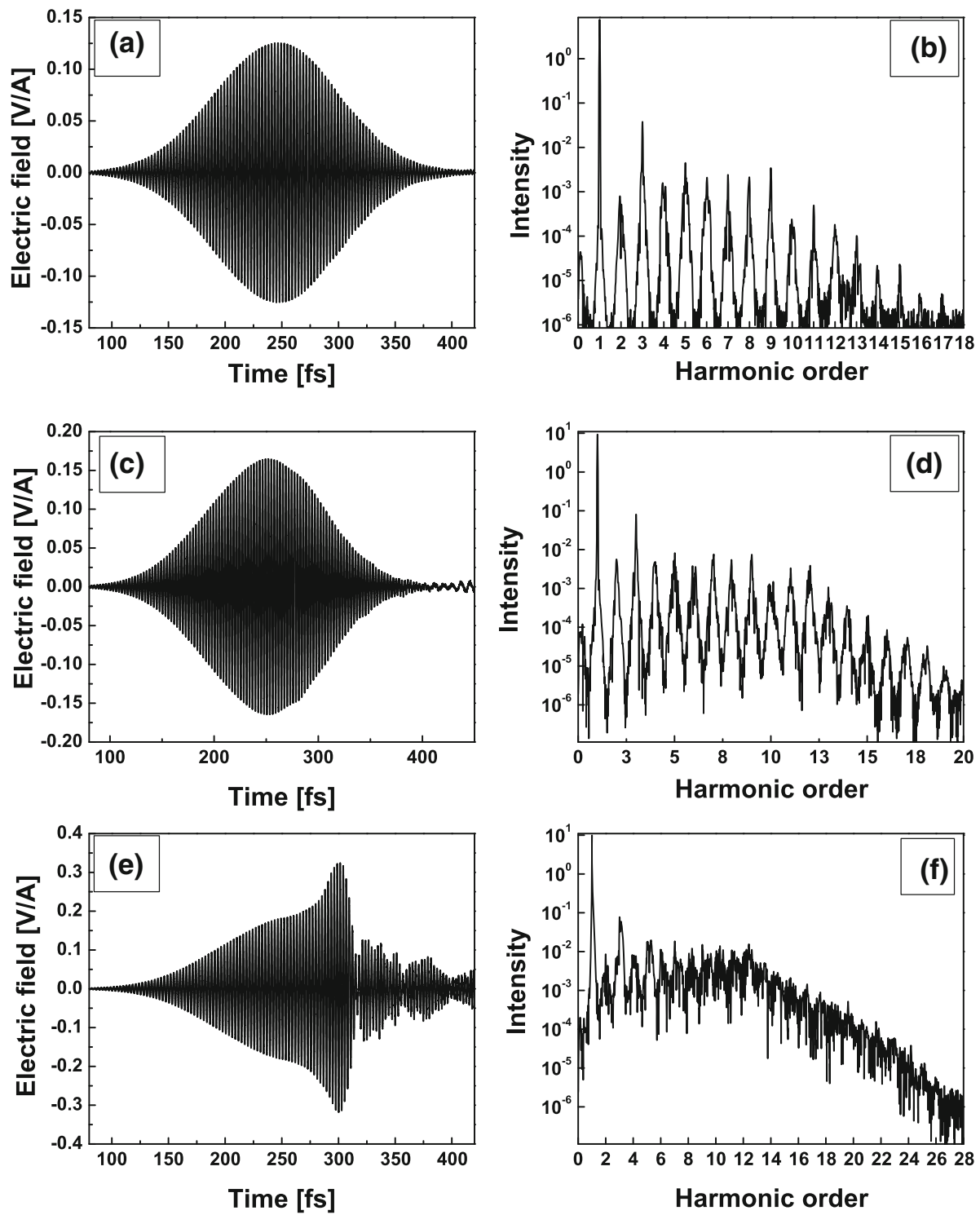

Fig. 4 Time evolution of the pulsed total electric field (in $\mathrm{V} / \AA$ ) in bulk silicon for $\tau=90$ fs. The laser intensity at the pulse peak is $3 \times 10^{13} \mathrm{~W} / \mathrm{cm}^{2}$ in $(\mathbf{a}), 4.5 \times 10^{13} \mathrm{~W} / \mathrm{cm}^{2}$ in $(\mathbf{c})$ and is $5 \times 10^{13} \mathrm{~W} / \mathrm{cm}^{2}$ in (e). The laser is linearly polarized along the [001] crystal direction, and the laser wavelength is $800 \mathrm{~nm}$. The corresponding harmonic spectra are shown in (b), (d), (f)
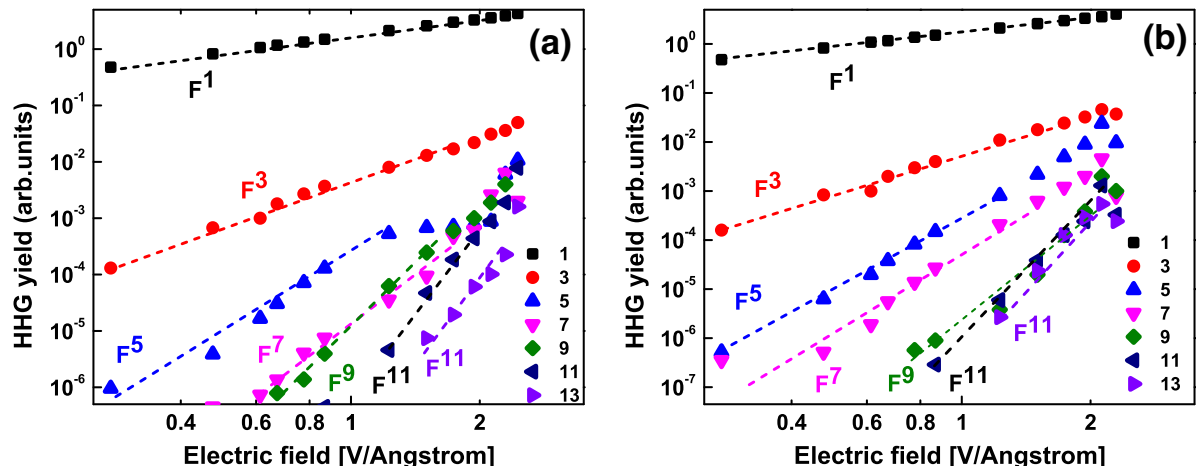

Fig. 5 Yield of high harmonics emitted in the silicon bulk as a function of the peak field strength for 30 fs laser pulse with wavelength of $800 \mathrm{~nm}$. The laser is linearly polarized along the [001] crystal direction in (a) and along the [111] crystal direction in (b) 

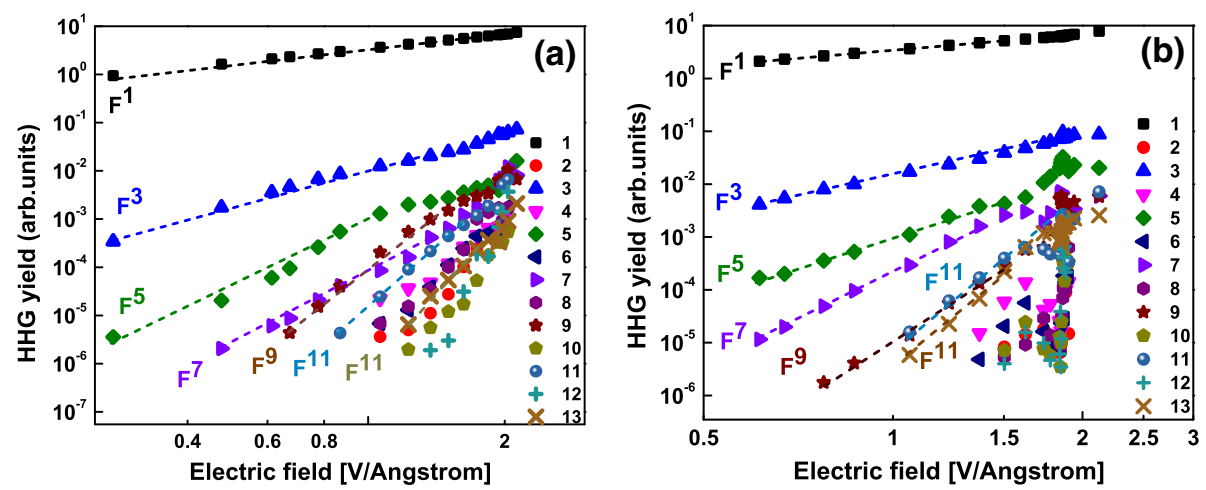

Fig. 6 Yield of high harmonics emitted in the silicon bulk as a function of the peak field strength for 60 fs laser pulse with wavelength of $800 \mathrm{~nm}$. The laser is linearly polarized along the [001] crystal direction (a), and along [111] crystal direction (b)
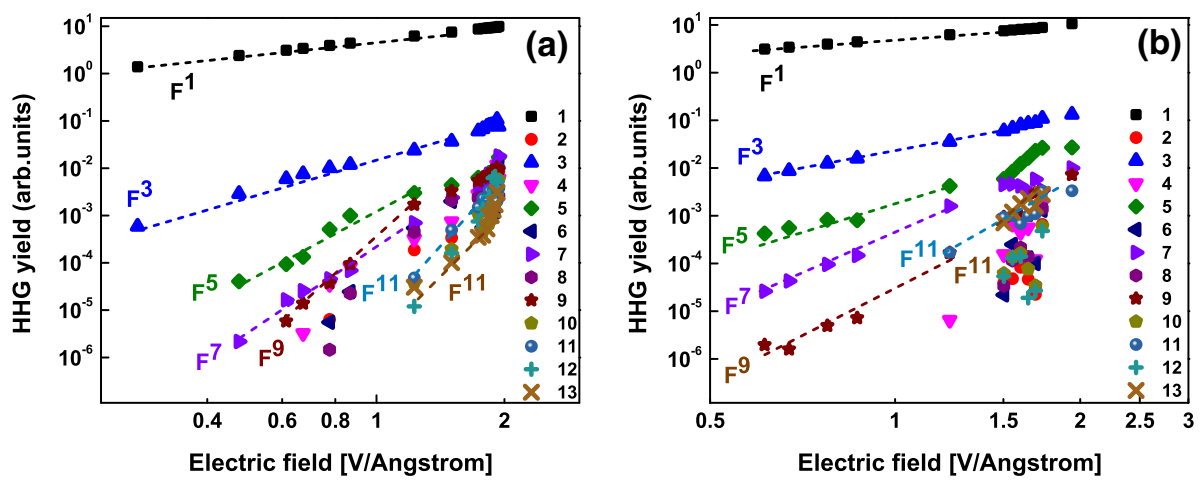

Fig. 7 Yield of high harmonics emitted in the silicon bulk as a function of the peak field strength for 90 fs laser pulse with wavelength of $800 \mathrm{~nm}$ and linear polarization along the [001] direction (a) and along the [111] (b)

much lower than the odd-order harmonic yield, which is in agreement with the prediction of the theory outlined in the previous section. When the field strength is increased to $1.5 \mathrm{~V} / \AA$, the yields of even and odd harmonics become comparable.

III. Breakdown regime with $F>2 \mathrm{~V} / \AA$ : the laser has ionized the target and high-density plasma of freecharge carriers is created. The plasma irreversible modifies the optical properties of the photo-excited silicon, the temporal coherence of the transmitted pulse decreases, and its temporal shape is modified by the laser-matter interaction. The transmitted pulse does not exhibit a quasi-DC component, and as a result, the emission of even-order harmonics is absent. In this regime, the spectral intensity of individual harmonics is indistinguishable.

\section{Conclusion}

In summary, we have investigated the ultrafast photoexcitation and high harmonic generation in crystalline silicon subjected to intense ultrashort near-infrared laser pulses in a wide range of peak laser intensities. For the considered range of laser intensities and pulse duration $\tau \leq 100 \mathrm{fs}$, high harmonic generation has low conversion efficiency of near-infrared to ultraviolet irradiation. For relatively low laser intensity, the harmonic spectra in silicon follow the prediction of the perturbative nonlinear optics: only odd-order harmonics are generated with intensities which scale as function of the peak laser intensity according to the law $I_{n} \sim I^{n}$. For moderate laser intensities and increased coherence length of the laser pulse $(c \tau \geq 18 \mu \mathrm{m}$, here $c$ is the speed of light), we find a non-perturbative effect of even harmonic generation. In this regime, the transmitted pulse undergoes optical rectification associated with generation of quasi-DC electric field as low as $2 \mathrm{~V} / \mu \mathrm{m}$, which affects the phase relationship between interfering alternatives for HHG during each two consecutive half-cycles of driving laser. The phase shift accumulated during propagation of electron-hole pairs in the DC field allows production of even harmonics. For high level of electronic excitation, when dielectric breakdown of silicon occurs, the temporal coherence of the transmitted laser pulse deteriorates; consequently, the harmonic spectra are blurred and a quasi-continuous spectrum emerges.

Acknowledgements Cost action CA17126 and support from the Bulgarian National Science Fund under Contract No. KP-06-KOST are acknowledged. 


\section{Author contributions}

Both authors contributed to the paper.

Data Availability Statement This manuscript has no associated data or the data will not be deposited. [Authors' comment: All results presented in this paper can be reproduced with the information provided in the manuscript. The authors can be contacted for further information.]

Open Access This article is licensed under a Creative Commons Attribution 4.0 International License, which permits use, sharing, adaptation, distribution and reproduction in any medium or format, as long as you give appropriate credit to the original author(s) and the source, provide a link to the Creative Commons licence, and indicate if changes were made. The images or other third party material in this article are included in the article's Creative Commons licence, unless indicated otherwise in a credit line to the material. If material is not included in the article's Creative Commons licence and your intended use is not permitted by statutory regulation or exceeds the permitted use, you will need to obtain permission directly from the copyright holder. To view a copy of this licence, visit http://creativecomm ons.org/licenses/by/4.0/.

\section{References}

1. S. Ghimire, A.D. DiChiara, E. Sistrunk, P. Agostini, L.F. DiMauro, D.A. Reis, Nat. Phys. 7, 138 (2011)

2. S. Ghimire, A.D. DiChiara, E. Sistrunk, G. Ndabashimiye, U.B. Szafruga, A. Mohammad, P. Agostini, L.F. DiMauro, D.A. Reis, Phys. Rev. A 85, 043836 (2012)

3. S. Ghimire, G. Ndabashimiye, A.D. DiChiara, E. Sistrunk, M.I. Stockman, P. Agostini, L.F. DiMauro, D.A. Reis, J. Phys. B 47, 204030 (2014)

4. O. Schubert, M. Hohenleutner, F. Langer, B. Urbanek, C. Lange, U. Huttner, D. Golde, T. Meier, M. Kira, S.W. Koch, R. Huber, Nat. Photon. 8, 119 (2014)

5. M. Wu, S. Ghimire, D.A. Reis, K.J. Schafer, M.B. Gaarde, Phys. Rev. A 91, 043839 (2015)

6. P.G. Hawkins, M.Y. Ivanov, V.S. Yakovlev, Phys. Rev. A 91, 013405 (2015)

7. T. Higuchi, C. Heide, K. Ullmann, H.B. Weber, P. Hommelhoff, Nature 550, 224 (2017)

8. G. Vampa, T.J. Hammond, N. Thire, B.E. Schmidt, F. Legare, C.R. McDonald, T. Brabec, D.D. Klug, P.B. Corkum, Phys. Rev. Lett. 115, 193603 (2015)

9. M. Hohenleutner, F. Langer, O. Schubert, M. Knorr, U. Huttner, S.W. Koch, M. Kira, R. Huber, Nature 523, $572(2015)$

10. Y.S. You, Y. Yin, Y. Wu, A. Chew, X. Ren, F. Zhuang, S. Gholam-Mirzaei, M. Chini, Z. Chang, S. Ghimire, Nat. Commun. 8, 724 (2017)
11. A. Schiffrin, T. Paasch-Colberg, N. Karpowicz, V. Apalkov, D. Gerster, S. Muhlbrandt, M. Korbman, J. Reichert, M. Schultze, S. Holzner, J.V. Barth, R. Kienberger, R. Ernstorfer, V.S. Yakovlev, M.I. Stockman, F. Krausz, Nature 493, 70 (2013)

12. M. Schultze, K. Ramasesha, C.D. Pemmaraju, S.A. Sato, D. Whitmore, A. Gandman, J.S. Prell, L.J. Borja, D. Prendergast, K. Yabana, D.M. Neumark, S.R. Leone, Science 346, 1348 (2014)

13. M. Garg, M. Zhan, T.T. Luu, H. Lakhotia, T. Klostermann, A. Guggenmos, E. Goulielmakis, Nature 538, 359 (2016)

14. G. Vampa, T.J. Hammond, M. Taucer, Xiaoyan Ding, X. Ropagnol, T. Ozaki, S. Delprat, M. Chaker, N. Thire, B.E. Schmidt, F. Legare, D.D. Klug, A. Yu Naumov, D.M. Villeneuve, A. Staudte, P.B. Corkum, Nat. Photon. 12, 465 (2018)

15. A. Sommer, E.M. Bothschafter, S.A. Sato, C. Jakubeit, T. Latka, O. Razskazovskaya, H. Fattahi, M. Jobst, W. Schweinberger, V. Shirvanyan, V.S. Yakovlev, R. Kienberger, K. Yabana, N. Karpowicz, M. Schultze, F. Krausz, Nature (London) 534, 86 (2016)

16. H. Tom, T. Heinz, Y. Shen, Phys. Rev. Lett. 51, 1983 (1983)

17. Vampa, T.J. Hammond, N. Thire, B.E. Schmidt, F. Legare, D.D. Klug, P.B. Corkum, Generation of High Harmonics from Silicon (2016). arXiv:1605.06345

18. G. Vampa, T.J. Hammond, N. Thire, B.E. Schmidt, F. Legare, C.R. McDonald, T. Brabec, P.B. Corkum, Nature 522, 462-464 (2015)

19. G. Vampa, B.G. Ghamsari, S. Siadat Mousavi, T.J. Hammond, A. Olivieri, E. Lisicka-Skrek, A. Yu Naumov, D.M. Villeneuve, A. Staudte, P. Berini, P.B. Corkum, Plasmon-enhanced high-harmonic generation from silicon. Nat. Phys. 13, 659 (2017)

20. G. Vampa, C.R. McDonald, G. Orlando, P.B. Corkum, T. Brabec, Phys. Rev. B 91, 064302 (2015)

21. S. Jiang, H. Wei, J. Chen, C. Yu, R. Lu, C.D. Lin, Phys. Rev. A 96, 053850 (2017)

22. T. Apostolova, B. Obreshkov, Diam. Relat. Mater. 82, 165-172 (2018)

23. T. Apsotolova, B. Obreshkov, Opt. Quant. Electron. 50, 408 (2018)

24. T. Apostolova, B. Obreshkov, I. Gnilitskyi, Appl. Surf. Sci. 519, 146087 (2020)

25. B. Obreshkov, T. Apostolova, J. Phys. Conf. Ser. 1571, $012012(2020)$

26. M.L. Cohen, T.K. Bergstresser, Phys. Rev. 141, 789 (1966)

27. N.A. Papadogiannis, P.A. Loukakos, S.D. Moustaizis, Opt. Commun. 166, 133-139 (1999) 